\title{
Nanotechnology in Medicine: Moving from the Bench to the Bedside
}

\author{
Patrick R. Hunziker ${ }^{\mathrm{a}^{\star}}$, Martin Stolz ${ }^{\mathrm{b}}$, and Ueli Aebi ${ }^{\mathrm{b}}$
}

\begin{abstract}
While living matter is composed of a large number of biological nanomachines, it has been recognized early in the history of nanotechnology that medicine could be a prime field for application. Now that nanotechnology has gone beyond its infancy, its mature arsenal of tools, methods and materials is ready for applications outside physics. While true clinical applications of nanotechnology are still practically non-existent at the current time, a significant number of promising medical projects is at an advanced experimental stage. Tools based on the atomic force microscope will not only allow improved imaging of living matter but can also serve as functional probes and will even serve as sensitive sensors for a broad range of molecules of medical interest. New immunological tests based on microcontact printing and microfluidics will significantly improve medical laboratory diagnosis. New materials, including nanotubes and fullerenes, nanocontainers and other self-assembled structures may improve mechanical properties and biocompatibility of implants and will allow new approaches in drug targeting.
\end{abstract}

Keywords: Arthroscopy · Atomic force microscopy · Biochemical testing · Drug targeting · Medical implants $\cdot$ Medicine $\cdot$ Nanoscience

\section{Life is Nanoscience in Action}

To see living organisms designed as very complex systems composed of nanomachines and built by 'programmed self-assembly' might well be the ultimate paradigm in a mechanistic understanding of life. Down at the scale of single molecules and supramolecular assemblies, there is no gap between the classical fields of chemistry, mechanics, biology, and even medicine. Motion generated by muscle contraction illustrates this fact well: Physics ( $c f$. electric potentials within a cell), physical chemistry ( $c f$. free intracellular calcium), biochemistry ( $c f$. myosin phosphorylation), mechanics ( $c f$. bending of the myosin heads during the crossbridge cycle) fail to explain

${ }^{*}$ Correspondence: PD Dr. med. P. Hunzikera aUniversity Hospital of Basel

Medical Intensive Care Unit

Petersgraben 5

$\mathrm{CH}-4031$ Basel

Tel.: +41612652525

Fax: + 41612655300

E-Mail: Patrick.Hunziker@unibas.ch

bM.E. Müller Institute for Structural Biology

Biozentrum

University of Basel

Klingelbergstrasse 70

$\mathrm{CH}-4056$ Basel motion adequately. In contrast, the view of, for example, muscle myofilament assembly being a nanomachine involving electrical, physicochemical, organic chemical, mechanical and many other aspects, opens the door to a more comprehensive and, at the same time, more rational understanding of this system. This view becomes even more true when trying to deal with the most fundamental 'building block' of all living organisms - the cell.

However, it was not until the development of the atomic force microscope (AFM) that an analytical tool became available which is capable of imaging, measuring and manipulating biological matter at the level of single molecules or even atoms. Indeed, the AFM has opened completely new vistas in the life sciences for analyzing biological matter at a resolution that has hitherto only been achieved by transmission electron microscopy (TEM). On the one hand, unlike TEM and classical and confocal light microscopy that can look 'inside' biological matter, the AFM is a tool for probing surfaces. On the other hand, the AFM has the unique capability to study biological matter in its native environment, thereby providing the opportunity to directly correlate structural with functional states, for example, at the level of single molecules, living cells, and even entire tissues [1-3]. Moreover, indentation-type (IT) AFM has opened the possibility to probe the mechanical properties of biological matter at different length scales, i.e. from the $\mathrm{nm}$ to the $\mu \mathrm{m}$ and even $\mathrm{mm}$ scale [4-6]. Most exciting, by succeeding in dissecting DNA from a particular section chromosome, it has become evident that the AFM tip may also be used as a nano-scalpel [7].

Taken together, not only does the AFM give us the 'eyes' for imaging biological matter from the $\mathrm{mm}$ to the $\mu \mathrm{m}$ and, ultimately, nm scale, but it also provides us with the 'fingers' to measure and manipulate biological matter at these different length scales. As might be gathered, the prospects of this unique device in fundamental research and for practical applications in biology and medicine are only limited by our imagination.

\section{Many Diseases Have Their Roots at the Nanoscale}

A large number of diseases are best understood as a malfunction of biological nanomachines. Genetic diseases such as 
myopathies can lead to impaired molecular interactions during the course of muscle myofilament assembly [8]. A viral infection, in turn, may be considered as an invasion of a complex system (a cell) by a simple nanomachine (a virus) that is able to reprogram the system. Frequently, cancer represents a malfunctioning of a nanosystem encompassing a gene, its transcription apparatus, and promoter and suppressor mechanisms that in concert drive the cell into an 'infinite loop' of uncontrolled selfreplication. Arteriosclerosis too was eventually traced back to a dysfunction at the single cell level, i.e. involving abnormal handling of some lipoproteins [9] and local inflammatory reactions.

\section{The Gap of Today's Medical Tech- nology Resides at the Nanoscale}

In recent years the structure and function of an ever-growing number of supramolecular and cellular nanomachines have been elucidated, and the role these play in disease progression is increasingly recognized. Despite this, when we look at the current diagnostic and therapeutic 'medical toolbox', we find a remarkable discrepancy. As illustrated in Fig. 1, whereas the fundamental biological processes that govern health and disease happen at the cellular and subcellular scale, i.e. ranging from a few micrometers to a few nanometers, today's medical tools were designed almost exclusively for a different length scale: Diagnostic tools either rely on measuring bulk concentrations of single molecules, or they make use of structures and gadgets that are typically at the millimeter to meter scale.

Similar objections hold true for therapeutic interventions: Drug therapy usually involves loading the entire body with a large number of drug molecules to treat a limited number of malfunctioning cells. As we all know, the lack of targeting inherent to systemic drug application may lead to various side effects. For example, anticancer drugs not only kill tumors, they also affect the gut (nausea, vomiting), the immune system (susceptibility to infection), the blood (anemia), and the skin (hair loss). Side effects may occur unexpectedly, and they may not simply put the patient at risk, they may also jeopardize drug companies, for example, as recently illustrated by Bayer after their new drug cerivastatin killed several patients.

\section{The Promise of Nanotechnology in Medicine}

Ever since nanotechnology appeared on the horizon, its bright prospects in medicine have been hailed enthusiastically [10]. To this end, a recent Medline literature search using the keywords 'nanotechnology or nanoscience or nanotubes or nanocapsules or nanoparticles' yielded more than 2000 hits out of 11 million medical references. However, this number shrunk to 70 when the keyword 'clinical' was added. Even these publications failed to document a single established medical in vivo use of nanotechnology. Instead, almost all of these 70 publications focused on in vitro results, prediction of future possibilities, or on experimental concepts. Despite this sobering finding, the arguments in favor of a bright future for nanotechnology in medicine are numerous.

\section{Nanotools}

The currently available 'nanotechnology toolbox' allows the attainment of atomic detail not only for imaging single molecules in their native state, but to also to probe their functional properties, and to manipulate them one by one. The technology developed for atomic force imaging has led to miniaturized devices that can function as local probes, biosensors and effectors that are very well suited for integration into instruments of such small size so that they can directly be brought to the defect site by minimally invasive surgical procedures. This evolving nanotechnology toolbox is slowly but definitely filling the gap between conventional medical instruments and tools that are on a cellular or even subcellular length scale.

\section{Nanomaterials}

Nanotechnology has produced novel materials such as nanotubes [11] and nanospheres [12] that feature amazing mechanical properties. It is evident that such nanomaterials will eventually improve the design and properties of implants where optimum mechanical strength and durability are critical, for example, in artificial heart valves and weight-bearing artificial hip and knee prostheses. Presently, nano-structured surfaces represent a very active field of research and development which may ultimately lead to improved biocompatibility of nanomaterials.

\section{Biomedical Laboratory Diagnostics at the Nanometer Scale}

Biomedical laboratory diagnosis plays a key role in today's health care. Most testing is done on a macroscopic scale, for example, in microtiter plates. Size reduction of biomedical lab tests has several advantages: Not only does it lead to a marked reduction of the sample volume needed for testing, but it also results in a marked reduction of (potentially expensive) reagents such as monoclonal antibodies. Last but not least, it may lead to a significant reduction in time consumption. Moreover, the ability of current nanotools to measure interaction

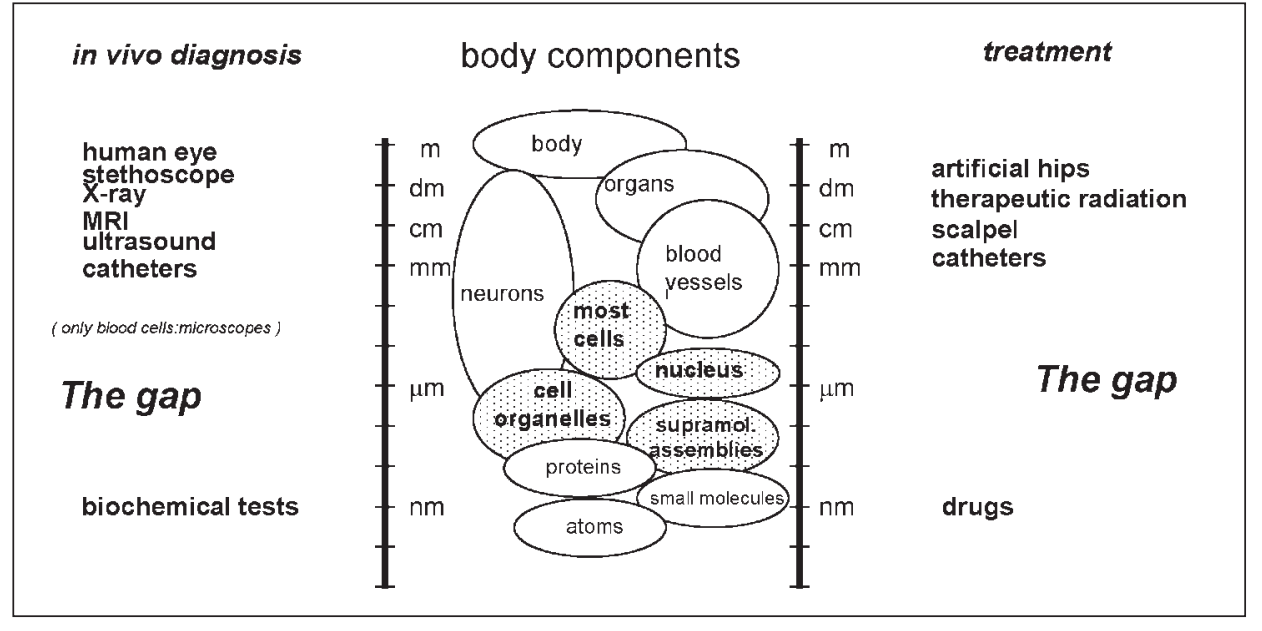

Fig. 1. In today's medicine, there is a lack of diagnostic and therapeutic tools applicable in vivo at the submicrometer scale - this is exactly the scale where many diseases originate. Nanotechnology promises to fill this gap. 
forces between individual molecules is most promising for biomedical testing because this might eliminate the need for reagent labeling, a tedious and expensive step. Taken together, small size, tiny sample volumes and fast reaction times bring mobile testing devices onto the realm of reality [13]. They indicate that there will be a strong trend toward point-of-care testing at the bedside or in an ambulatory setting.

\section{Examples of Nanotechnology in Medicine}

Following are three practical examples taken from our own research activities that will illustrate the application of nanotechnology in experimental medicine.

\section{Diagnosing Articular Cartilage Elasticity ex vivo by Indentation-type (IT) AFM}

Cartilage spans the articulating surfaces of bones where they form joints, such as knees, hips, elbows, shoulders, and the spine. The biomechanical properties of articular cartilage are such that under normal conditions it will stay intact for 70 or more years. Pathological situations such as acute trauma or chronic inflammation may lead to the degradation of the articulating surfaces in the affected joints [14].

Articular cartilage can be regarded as a fiber-reinforced multi-component and multi-phase material whose mechanical properties are governed by the complex architecture of the extra-cellular matrix (ECM). The most comprehensive information about the mechanical function of articular cartilage tissue can be obtained by measuring its elastic properties [15-18]. Therefore, we have been employing IT-AFM to directly measure the elastic modulus of healthy, diseased (e.g. osteoarthritic) and enzymedigested articular cartilage.

In general, representative elasticity measurements of a given piece of cartilage can be obtained by employing indenters that probe the tissue on a length scale (i.e. $\mathrm{mm}$ to $\mu \mathrm{m}$ ) that cannot resolve local fine structure, so within this length range the measurement does not exhibit a significant dependence on the probe size. To perform elasticity measurements at the micrometer scale we glued micrometer-sized spheres onto tipless cantilevers. As expected, the elasticity of articular cartilage determined at this length scale was in accordance with published values determined by clinical indentation devices, i.e. clinical testing devices that probe the elasticity of articular cartilage at the millimeter to centimeter scale.
For comparison, elasticity measurements obtained by indentation-type (IT) $\mathrm{AFM}$ at the nanometer scale (i.e. by employing sharp pyramidal tips) yielded elasticity moduli that were typically 100 - to 500-fold lower. Fig. 2a demonstrates the topography at the overall tissue level of articular cartilage as imaged by AFM. This image demonstrates what a micrometersized indenter 'feels' or 'sees' as the direct mechanical response at this level of the cartilage architecture. In contrast, Fig. 2b demonstrates a distinct substructure. At the nanometer scale distinct components, such as individual fibers, of the extracellular matrix (ECM) become visible. An individual collagen fiber exhibits a $67 \mathrm{~nm}$ axial repeat and $50 \mathrm{~nm}$ diameter.

In a next step, we compared normal with osteoarthritic articular cartilage from a patient harvested during hip surgery. As illustrated in Fig. 3a, the normal cartilage exhibits a more or less random orientation of the collagen fiber network, with the $67 \mathrm{~nm}$ axial repeat of the individual collagen fibers being clearly resolved. In contrast, the diseased cartilage exhibits a preferred orientation of the collagen fibers (Fig. 3b). It is conceivable that upon degradation of the glycosaminoglycans (GAGs) in the interstitial space, the collagen fibers that are no longer spaced apart by the GAGs slowly but definitely align themselves in a direction representing the predominant joint movement. As demonstrated in Fig. 3c, the osteoarthritic cartilage revealed a significant hardening compared to the normal cartilage when measured at the nanometer scale.
In further experiments, we mimicked the osteoarthritic disease progression by enzymatic digestion of the GAGs with cathepsin D. At the micrometer scale, i.e. when monitored with our spherical tips, no significant hardening was depicted (Fig. 4a), and the elastic modulus deduced from these measurements was in accordance with that obtained by macroscopic indentation tests. In contrast, at the nanometer scale the enzymatic digestion exhibited a significant hardening of the digested cartilage (Fig. 4b), exactly as observed with osteoarthritic cartilage (see Fig. 3c). Accordingly, the elastic modulus increased from $21 \mathrm{kPa}$ for the native articular cartilage to $54 \mathrm{kPa}$ after two days of enzyme action.

As expected, the elasticity values are dependent on the chemical composition and the spatial arrangement of the collagen network. At the nanometer scale the fine structure of cartilage is apparent, i.e. on the collagen fibers and the space in-between (i.e. the interstitial space being filled with glycosaminoglycans (GAGs) keeping adjacent collagen fibers spaced apart). By removing some of the GAGs through enzymatic digestion with cathepsin $\mathrm{D}$ the collagen fibers became more densely packed thereby resulting in hardening of the digested cartilage at the nanometer scale. Two results are most important in this context: First, the digestion experiments allowed us to model or mimic alterations similar to those caused by the pathological degradation processes. Secondly, the elasticity measurements as provided at two different scales (i.e. nanometer versus micro-

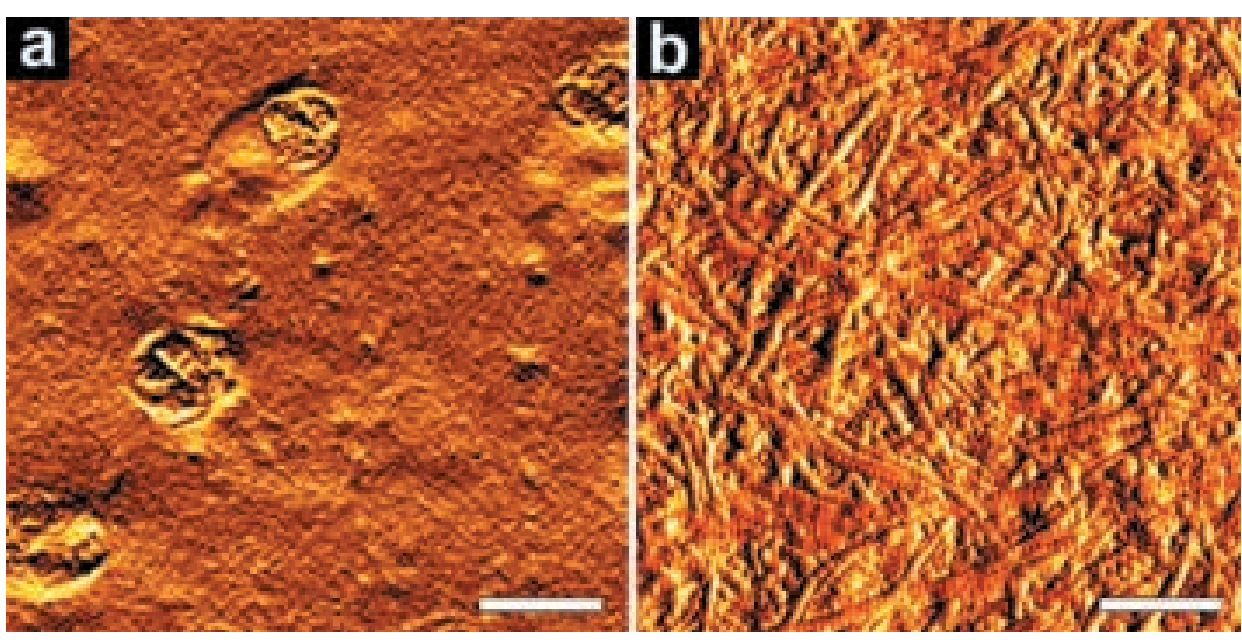

Fig. 2. The two images demonstrate two different levels of the cartilage architecture as imaged by AFM. (a) Exhibits the overall tissue level, where the tissue functions as a compact multicompound system. At this level of hierarchy distinct cartilage cells (chondrocytes) and the compact extracellular matrix (ECM) are visible, but no distinct sub-structure of the ECM. (b) At the nanometer scale the cartilage tissue exhibits its substructure. At this level the randomly ordered collagen fibers, i.e. the $67 \mathrm{~nm}$ axial repeat and $50 \mathrm{~nm}$ diameter of the individual collagen fibers as distinct components of the ECM are clearly visible. Scale bars: (a) $20 \mu \mathrm{m}$, (b) $1 \mu \mathrm{m}$. 

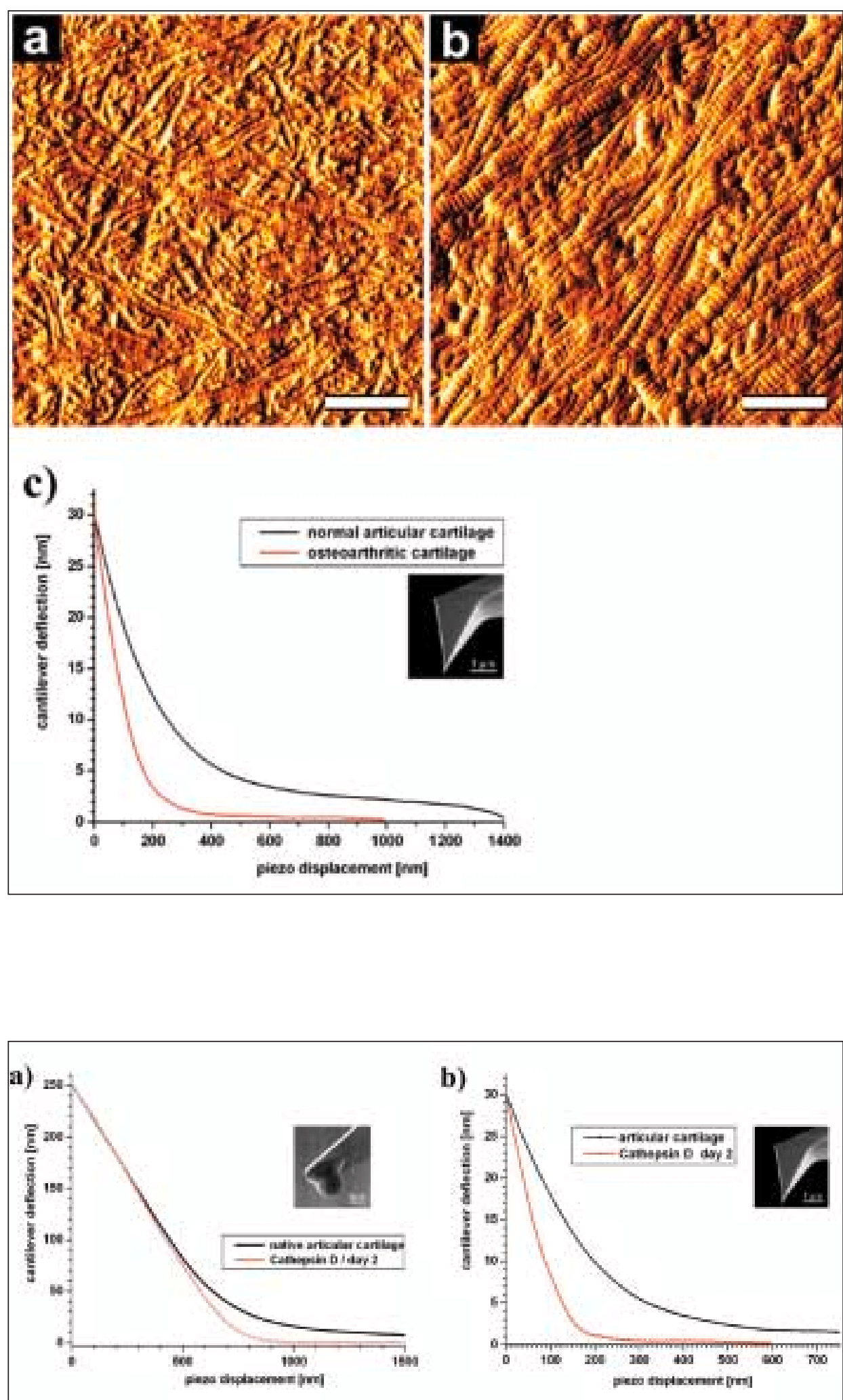

Fig. 4. The elasticity measurements as presented were taken from the same samples by employing two different indenter sizes, i.e. (a) micrometer-sized spherical tips (diameter of the tip $=5$ micrometer) and (b) nanometer-sized pyramidal shaped sharp tips (nominal tip-radius $\leq 2 \mathrm{~nm}$ ). Each set of the two curves is taken on normal articular cartilage and on cathepsin D digested articular cartilage. For digestion the articular cartilage was treated by cathepsin $\mathrm{D}$ at $37^{\circ} \mathrm{C}$ for $2 \mathrm{~d}$. Comparisons of the two slopes as taken at the micrometer scale indicate no difference between native and cathepsin $D$ treated articular cartilage. At the nanometer scale the situation is different, i.e. the cathepsin D treated articular cartilage exhibits a clear stiffening. The two insets suggest at what level the indenters assess the cartilage architecture, i.e. a micrometer scale indenter may distinguish between the chondrocytes and the ECM, whereas at the nanometer scale the distinct fine-structure of the ECM, such as the interstitial space between the collagen fibers being filled with glycosaminoglycans (GAGs) that often is altered in a disease progression can be assessed by the small indenters.
Fig. 3. Surface topography of (a) normal articular cartilage and (b) osteoarthritic articular cartilage, imaged in air and (c) the corresponding elasticity measurement at the nanometer scale. (a) The $67 \mathrm{~nm}$ repeat of individual collagen fibers is clearly resolved by AFM. (b) In contrast to the normal cartilage that exhibits a random orientation of the collagen network, in the diseased cartilage the collagen fibers exhibit a preferred orientation. The orientation of the fibers might follow the directed movement within the joint more easily once the GAGs become digested in the course of the disease development. (c) Comparison of the two slopes indicates stiffening of the osteoarthritic articular cartilage. Scale bars: (a and b) $1 \mu \mathrm{m}$.

meter) resulted in qualitatively different results, i.e. the mechanical properties of articular cartilage can be very different depending on whether they are measured at the micrometer or the nanometer scale.

\section{Microcontact Printing, Microfluidic Networks, and Micromosaic Immunoassays}

Five-hundred years after Gutenberg printed his first bible, printing technology has been rejuvenated by the recent development of microcontact printing and related techniques including micromosaic immunoassays [19-21]. Microcontact printing is based on the observation that some polymers can be molded to a very small feature size. When coated with an appropriately diluted sample, e.g. containing proteins in solution, they can act as a stamp to print its features on a surface. Microcontact printing of proteins down to single molecules has been reported. This, in turn, allows the design of immunoassays at very small scales, bringing all the mentioned advantages including the marked reduction of sample and reagent volume needed combined with rapid processing times and parallel processing of multiple samples. Alternatively, reagents can be brought to the test substrate surface by microfluidic networks that consist of channels with diameters in the micrometer range. Flow of samples and analytes is driven by capillary forces or by miniature pumps. The molecules to be tested are thus brought to the testing site primarily by convection, while diffusion (the time limiting step in conventional assays) is necessary only across a distance measured in micrometers, not in millimeters, rendering the tests very fast. In our prototype tests, overall processing times in the range of 5-10 minutes were realistic even without automated equipment. 
Fig. 5 illustrates such a micromosaic immunoassay based on microfluidics. Note that the actual test surface per sample measures $1 / 2500$ square millimeter, compared to several square millimeters in conventional enzyme linked immunoassays (ELISA) in microtiter plates. With such small test surfaces, we actually found that reliable tests can be done with sample volumes far below one microliter. Size reduction also is an opportunity to perform a large number of tests in parallel, which is an important prerequisite in the coming proteomics era in health care.

\section{Nanocontainers Used as Drug Delivery Vehicles}

Because systemic application of potent drugs carries a well-known risk of toxicity, local drug delivery to specific target organs or cells is an old dream. While direct injection and local intraarterial infusion are rarely used for various reasons, liposomes have raised considerable interest in the past two decades, especially when functionalized with specific ligands or antibodies. However, liposomal drug targeting remains of limited value in clinical medicine today, in part because of the limited stability and design flexibility liposomes can offer. In contrast, polymer nanocontainers [22][23] have not only a much increased stability but give the designer a lot of freedom in engineering: Successful integration of functional biological membrane proteins into the containers has been described by several groups. Based on these observations, functional nanocontainers have been constructed that are able to respond to specific external stimuli via their membrane receptors, while creating a compartment that can be used as a reaction chamber for local chemical reactions. This flexibility of nanocontainers makes them a prime candidate for targeted drug delivery, although many questions, including those of biocompatibility, toxicity, and immunogenicity are not exhaustively answered at the current time.

Because macrophages play a major role in a wide range of disease including cancer, immunologic, and cardiovascular disease, we chose macrophage cell lines as one model system for evaluation of new drug targeting approaches. On the surface of activated macrophages, scavenger receptors [24] are expressed that are responsible for binding and uptake of highly polyanionic molecules. As illustrated in Fig. 6a, nano- containers built from PMOXA-PDMSPMOXA triblock-copolymer (Graff et al, University of Basel) were specifically functionalized with poly- $G$ chains that form highly polyanionic DNA quadruplexes [25] and are well-known ligands of the scavenger receptor. Specific binding to the scavenger receptor could be proven by colocalisation experiments in human monocyte-derived macrophages, as well as in a tumor cell line mimicking macro-phages. Nanocontainers were then tagged with a fluorescent marker which allowed to track the cellular uptake of this prototype drug carrier (Fig. 6b). Current experiments focus on different release mechanisms of drugs contained in these 'Trojan horses' and on the specific impact of different drugs on the cells.

\section{Outlook}

Nanoscience, best thought of as an interdisciplinary field combining many of the classical natural sciences, offers a fresh look on life as a complex system composed of a large number of nanomachines. The fascinating and versatile toolbox offered by

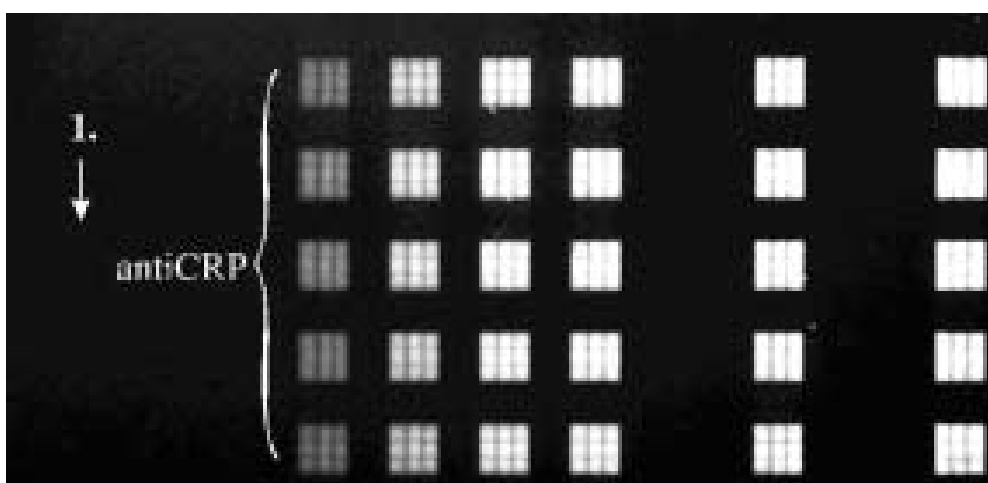

antiMyoglobin

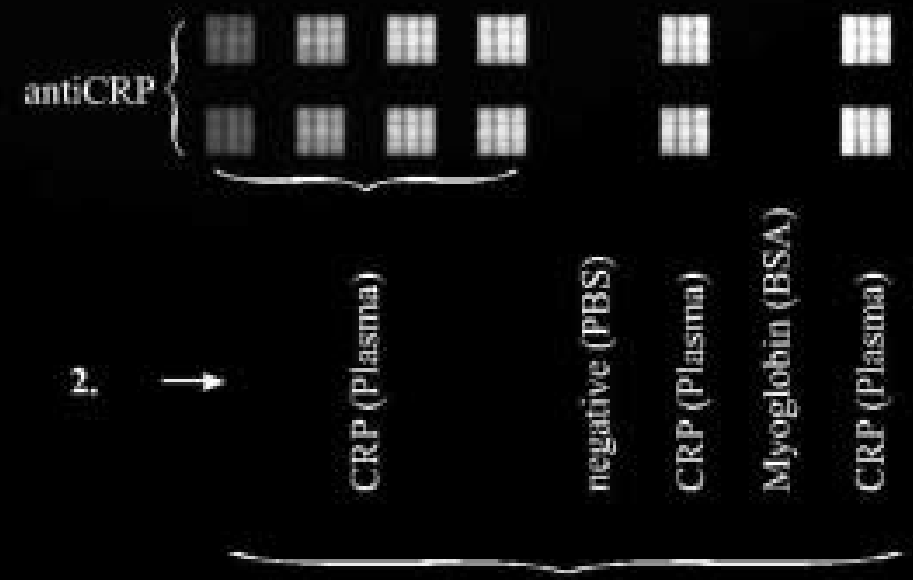

3.

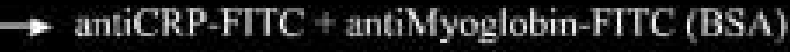

Fig. 5. Micromosaic immunoassays based on microfluidics allow parallel fast testing of multiple antigens in clinical samples within a few minutes with tiny amounts of reagents at a very small scale. This example shows a novel high-sensitivity test for $\mathrm{C}$-reactive protein, an important marker of inflammation in clinical practice. Silicon-chip based microfluidic networks were used for deposition of capture antibodies on a substrate, followed by sample application in a microfluidic network arranged perpendicularly, with final use of a fluorescent secondary antibody. Note that a single test area covers no more than $1 / 2500$ square millimeter, can be performed reliably with sample volumes in the range of 100 nanoliters, and can be performed within minutes. Image: Marc Wolf, IBM Rueschlikon and University Hospital Basel. 
nanotechnology is about to find a prime application in life sciences. Nanotechnology will advance today's medicine in two ways: First by improving current technology, e.g. by offering new materials. This will probably be the main impact in medicine in the short term. Secondly, we believe that the unique characteristics of nanotechnology will allow to break completely new ground through very innovative approaches focusing diagnosis and treatment on the single cell; this may happen later, but could have an even stronger impact on the way medicine is performed in the future.

The compelling advantages of nanotechnology applied to biomedical lab testing will bring a breakthrough in this field within a few years and will strongly enhance fast, bedside testing of whole arrays of parameters aiding in fast and accurate diagnosis.

Very flexible and highly selective drug targeting using nanocontainers will be applicable in selected applications within a few years and will have a big potential for development, including novel strategies for somatic gene therapy. Nanocontainers might also form the platform for simple nanosystems for medical use that combine sensing and effector functions at a submicrometer scale, although these systems will be far from what is considered a nanorobot by current science fiction descriptions.

For future clinical applications, our ex vivo measurements of articular cartilage elasticity by IT-AFM call for moving this diagnostic tool from the lab to the patient, i.e. by bringing the AFM directly into, for example, the knee joint using minimally invasive surgical procedures such as arthroscopy. Fig. 7 shows a cartoon as to how an 'arthroscopic' AFM might look like so that it can directly be introduced, i.e. through an arthroscopic tubus, into a knee joint, where it has to be stabilized relative to the joint surface to be analyzed.

Nanotools brought down to a scale that fits on a catheter tip will allow disease to be monitored and diagnosed in a minimally invasive way within the body within several years.

Fig. 7. Cartoon of an AFM designed for arthroscopic inspection of the articular cartilage in a knee joint. The arthroscopic AFM has been designed by S. Martin and H.J. Hug from the Physics Department, University of Basel, in collaboration with U. Stauffer from the Institute for Microtechnique, University of Neuchatel. The images of the knee joint are courtesy of Castle Orthopaedics, Auora, III, USA.

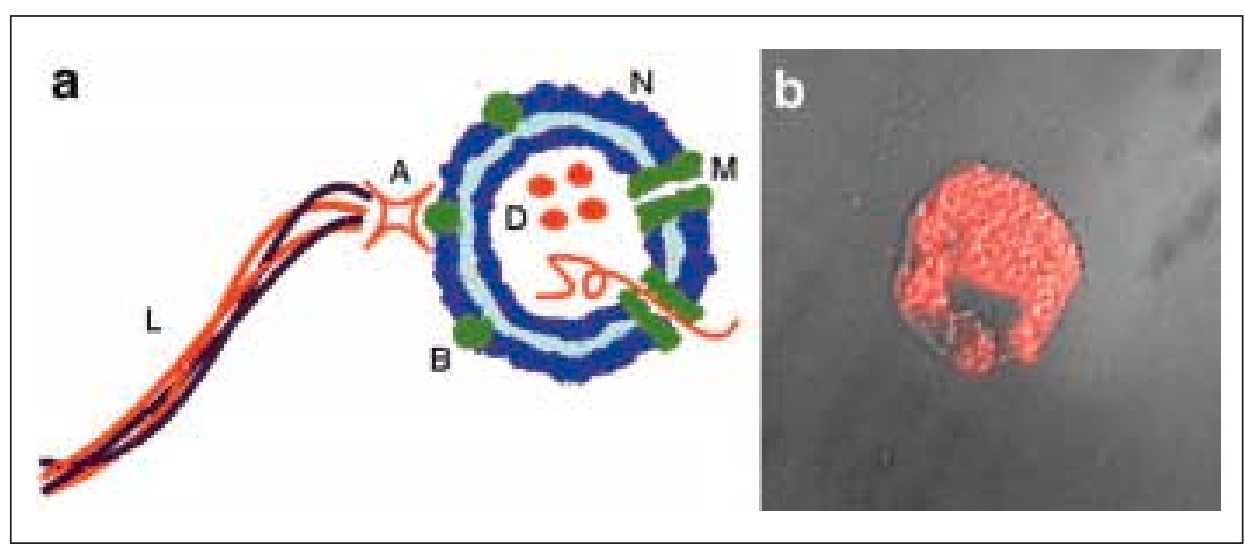

Fig. 6. Drug targeting by functionalized nanocontainers: (a). ABA triblock-copolymer nanocontainers $[N]$, functionalized through a biotin $[B]$-avidin $[A]$ link with poly-G quadruplexes $[\mathrm{L}]$, a ligand of macrophage scavenger receptors. Nanocontainers were filled with a fluorescent dye [D] and used as a prototype drug delivery device in human monocyte-derived macrophages. Optionally, these nanocontainers can be equipped with transmembrane proteins, allowing to expand functionality quite freely [M]. (b) Fluorescent labeled nanocontainers are specifically taken up by the target cells exposing scavenger receptors through endocytosis, where the drug content of the nanocontainer shall be released, in our case resulting in strong intracellular fluorescence. Specific binding to the receptor is documented in colocalisation experiments (not shown). This way, a systemic application of a potentially toxic drug can be circumvented, and a drug can be directly delivered to a specific class of target cells. Images: Cheelong Saw, University Hospital Basel/University of Basel.

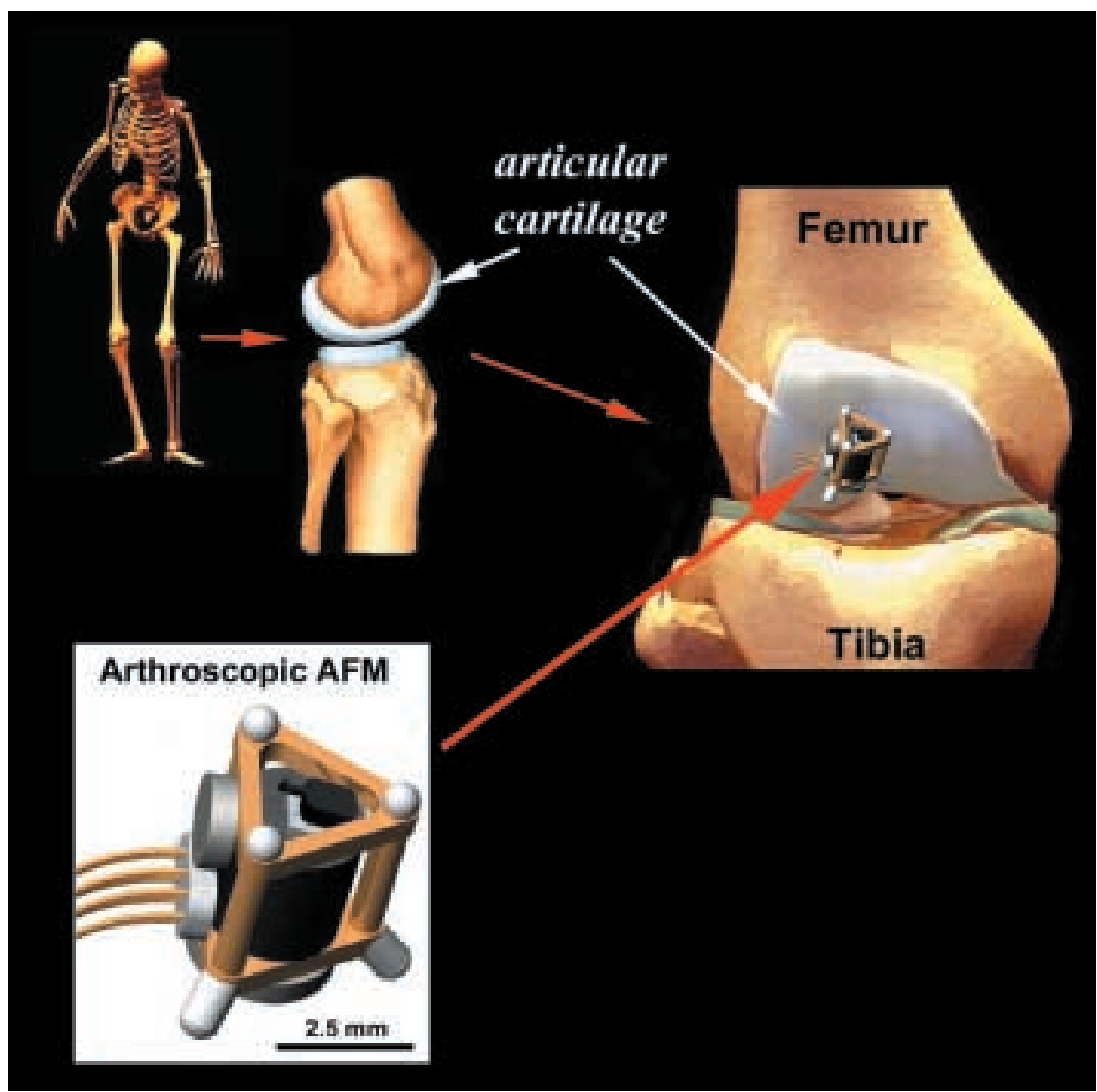


Single molecule detection, single cell diagnosis, and single cell treatment could become feasible within a decade.

While placative uses of nanotechnology like improvement of brain function and prolongation of lifespan have been declared as goals of medical nanotechnology research, we believe that in order to have a major impact on health, our research should focus on prevention and early treatment of the big killers: cardiovascular disease, cancer, and, in less developed countries, infections. Occasions for specific niche applications targeted to diseases with a limited number of patients will arise as research is ongoing.

Received: September 4, 2002

[1] D. Stoffler, K.N. Goldie, B. Feja, U. Aebi, 'Calcium-mediated structural changes of native nuclear pore complexes monitored by time-lapse atomic force microscopy', J. Mol. Biol. 1999, 287, 741-752.

[2] C. Goldsbury, J. Kistler, U. Aebi, T. Arvinte, G.J. Cooper, 'Watching amyloid fibrils grow by time-lapse atomic force microscopy', J. Mol. Biol. 1999, 285, 33-39.

[3] M. Stolz, D. Stoffler, U. Aebi, C. Goldsbury, 'Monitoring biomolecular interactions by time-lapse atomic force microscopy', J. Struct. Biol. 2000, 131, 171-180.

[4] E. A-Hassan, E., W.F. Heinz, M.D. Antonik, N.P. D'Costa, S. Nageswaran, C.A. Schoenenberger, J.H. Hoh, 'Relative microelastic mapping of living cells by atomic force microscopy', Biophysical Journal 1998, 74(no.3; March), 1564-1578.

[5] C. Rotsch, M. Radmacher, 'Drug-induced changes of cytoskeletal structure and mechanics in fibroblasts: an atomic force microscopy study', Biophysical Journal 2000, 78(no.1, pt.1; Jan.), 520-535.

[6] S. Hengsberger, A. Kulik, P. Zysset, 'Nanoindentation discriminates the elastic properties of individual human bone lamellae under dry and physiological conditions', Bone 2002, 30, 178-184.

[7] S. Thalhammer, R.W. Stark, S. Muller, J. Wienberg, W.M. Heckl, 'The atomic force microscope as a new microdissecting tool for the generation of genetic probes', $J$. Struct. Biol. 1997, 119, 232-237.

[8] K.J. Nowak, D. Wattanasirichaigoon, H.H. Goebel, M. Wilce, K. Pelin, K. Donner, R.L. Jacob, C. Hubner, K. Oexle, J.R. Anderson, C.M. Verity, K.N. North, S.T. Iannaccone, C.R. Muller, P. Nurnberg, F. Muntoni, C. Sewry, I. Hughes, R. Sutphen, A.G. Lacson, K.J. Swoboda, J. Vigneron, C. Wallgren-Pettersson, A.H. Beggs, N.G. Laing, 'Mutations in the skeletal muscle alpha-actin gene in patients with actin myopathy and nemaline myopathy', Nat. Genet. 1999, 23, 208-212.

[9] V.L. Herrera, S.C. Makrides, H.X. Xie, H. Adari, R.M. Krauss, U.S. Ryan, N. RuizOpazo, 'Spontaneous combined hyperlipi- demia, coronary heart disease and decreased survival in Dahl salt-sensitive hypertensive rats transgenic for human cholesteryl ester transfer protein', Nat. Med. 1999, 5, 1383-1389.

[10] K.E. Drexler, 'Engines of creation', Anchor Books, 1986.

[11] A. Thess, R. Lee, P. Nikolaev, H. Dai, P. Petit, J. Robert, C. Xu, Y.H. Lee, S.G. Kim, A.G. Rinzler, D.T. Colbert, G.E. Scuseria, J.E. Fischer, R.E. Smalley, 'Crystalline Ropes of Metallic Carbon Nanotubes', Science 1996, 273, 483-487.

[12] H.W. Kroto, J.R. Heath, S.C. O'Brien, R.F Curl, R.E. Smalley, ' $\mathrm{C}_{60}$ Buckminsterfullerene', Nature 1985, 318(no.6042; 14 Nov. 1985), 162-163.

[13] M.K. Baller, H.P. Lang, J. Fritz, C. Gerber, J.K. Gimzewski, U. Drechsler, H. Rothuizen, M. Despont, P. Vettiger, F.M. Battiston, J.P. Ramseyer, P. Fornaro, E. Meyer, H.J. Guntherodt, 'A cantilever array-based artificial nose', Ultramicroscopy 2000, 82(no.1-4; Feb. 2000), $1-9$.

[14] C.A. McDevitt, H. Muir, 'Biochemical changes in the cartilage of the knee in experimental and natural osteoarthritis in the dog', J. Bone Joint Surg. Br. 1976, 58, 94-101.

[15] C.G. Armstrong, V.C. Mow, 'Variations in the intrinsic mechanical properties of human articular cartilage with age, degeneration, and water content', J. Bone Joint Surg. Am. 1982, 64, 88-94.

[16] N.P. Cohen, R.J. Foster, V.C. Mow, 'Composition and dynamics of articular cartilage: structure, function, and maintaining healthy state', J. Orthop. Sports Phys. Ther. 1998, 28, 203-215.

[17] V. Roth, V.C. Mow, 'The intrinsic tensile behavior of the matrix of bovine articular cartilage and its variation with age', J. Bone Joint Surg. Am. 1980, 62, $1102-1117$.

[18] P.X. Ma, B. Schloo, D. Mooney, R. Langer, 'Development of biomechanical properties and morphogenesis of in vitro tissue engineered cartilage', J. Biomed. Mater. Res. 1995, 29, 1587-1595.

[19] A. Bernard, E. Delamarche, H. Schmid, B. Michel, H.R. Bosshard, H. Biebuyck, 'Printing patterns of proteins', Langmuir 1998, 14(no.9; 28 April), 2225-2229.

[20] A. Bernard, B. Michel, E. Delamarche, 'Micromosaic immunoassays', Anal. Chem. 2001, 73, 8-12.

[21] B. Michel, A. Bernard, A. Bietsch, E. Delamarche, M. Geissler, D. Juncker, H. Kind, J.P. Renault, H. Rothuizen, H. Schmid, W.P. Schmidt, R. Stutz, H. Wolf, 'Printing meets lithography: soft approaches to high-resolution printing', IBM Journal of Research and Development. 2001, 45(no.5; Sept), 697-719.

[22] M. Sauer, W. Meier, D. Streich, 'pH-sensitive nanocontainers', Advanced Materials 2001, 13, 1649-1651.

[23] A. Graff, M. Sauer, P. Van Gelder, W. Meier, 'Virus-assisted loading of polymer nanocontainer', Proc. Natl. Acad. Sci. USA 2002, 99, 5064-5068.
[24] M. Krieger, J. Herz, 'Structures and functions of multiligand lipoprotein receptors: Macrophage scavenger receptors, and LDL receptor-related protein (LRP)', $A n$ nu. Rev. Biochem. 1994, 63, 601-637.

[25] T. Simonsson, 'G-quadruplex DNA structures - variations on a theme', Biol Chem. 2001, 382, 621-628. 